\title{
Effects of Physical Activity on Memory Deficits Occurring Subsequent to Hypoxic Encephalopathy: a Single-Case Study*
}

\author{
Maki Kojima', Junji Nakamura' ${ }^{1,2}$, and Akinori Nagano ${ }^{3}$ \\ ${ }^{1}$ Department of Rehabilitation Medicine, Nishiyamato Rehabilitation Hospital \\ 3-2-2 Sasayuri-dai, Kanmaki-cho, Kitakatsuragi-gun, Nara 639-0218, Japan \\ Maki Kojima makiyama.sun@gmail.com \\ ${ }^{2}$ Graduate School of Health Science, Kio University \\ 4-2-2 Umaminaka, Koryo-cho, Kitakatsuragi-gun, Nara 635-0832, Japan \\ ${ }^{3}$ Faculty of Sport and Health Science, Ritsumeikan University \\ 1-1-1 Nojihigashi, Kusatsu-shi, Siga 525-8577, Japan \\ *Original article published in Japan J. Phys. Educ. Hlth. Sport Sci. 63: 827-836, 2018 (in Japanese) \\ [Received February 27, 2019; Accepted May 18, 2019; Published online May 28, 2019]
}

\begin{abstract}
Various methodologies have been employed for memory rehabilitation. However, inappropriateness of these approaches for patients suffering from marked memory deficit necessitates new effective approaches. Although studies report that physical activity and exercise can affect cognitive function, they lack sufficient empirical evidence. Therefore, we aimed to elucidate the effect of aerobic training on memory ability.

The subject was a 48-year-old, right-handed man with memory deficit subsequent to hypoxic encephalopathy. We used an A-B-A single-case experimental design. The subject performed delayed word-recall task and word fluency task 10 times in each phase. During the baseline Aand washout A-phases, after memorizing 3 words, the subject performed a paper and pencil task for 15 minutes, and thereafter recalled the 3 memorized words and performed the word fluency task. During the B-phase, after memorizing 3 words, the subject pedaled a bicycle ergometer at an intensity of $50 \mathrm{~W}$ for 15 minutes, and thereafter performed the word-recall and word fluency tasks.

Average performance in the delayed recall task was $0 \pm 0$ words in the baseline A-phase, $2.3 \pm$ 1.1 words in the B-phase, and $0.1 \pm 0.3$ words in the washout $A$-phase $(F(2,18)=37.098, p<$ 0.0001 ). The corresponding values in the word fluency task were $2.7 \pm 0.9,2.3 \pm 1.3$, and $3.6 \pm$ 1.3 words, respectively.

These results suggest that aerobic training can lead to the recovery of memory deficit. Although acute effects were observed, comprehensive recovery of cognitive function was not achieved.
\end{abstract}

Keywords: cerebrovascular disease, amnesia, aerobic training, cognitive function, rehabilitation

\section{Introduction}

A memory disorder occurs due to cerebral lesions, which have multiple etiologies such as head injury, stroke, epilepsy, multiple sclerosis, etc. Symptoms have been reported to develop in one-third of the patients who have had a stroke (Doornhein et al., 1998). However, functional restoration is difficult to achieve. Furthermore, rehabilitation will be difficult with regard to a patient's independence or social participation (Tatemichi et al., 1994).
For the rehabilitation of a memory disorder, approaches such as restitution of an environmental manipulation and a learning method, use of a compensatory tool, and group training have mainly been advocated so far (Watamori et al., 2005). These methods are employed in a theoretical framework, to promote a patient's active attendance, after combining with a tailor-made method based on the severity of each case or the characteristic of impairment (Mimura et al., 2003). Although reports on the usefulness of these approaches are plentiful, 
the unsuitability of these approaches for a person with a serious memory disorder has been pointed out (Cicerone et al., 2011). Therefore, the development of a more effective tool for the restitution of memory is expected.

The relationship of body activity and physical exercise with cognitive function has been reported in the recent years. A report showed that the attention, processing speed, executive function, and memory function of elderly's effectively improved due to aerobic exercise (Smith et al., 2010). Furthermore, a study reported that the reduction of cognitive function was suppressed in the trial candidate programmed the aerobic exercise compared to the group in which the aerobic training was not carrying out (Colcombe et al., 2004, Kramer et al., 2006). In patients with cerebrovascular diseases (CVD), a significant relationship was observed between a convalescent patient's total energy expenditure and the cognitive function item of Functional Independence Measure (FIM) (Kojima and Nagano, 2018). A longitudinal study reported that physical activity deterred the progression of dementia (Aarsland et al., 2010); aerobic exercise improved the performance and processing speed, as measured by Serial reaction time task (SRTT), in patients with chronicstage stroke, compared to the control group (Quaney et al., 2009). Furthermore, after exercising recreationally for six months, memory and executive function were reported to improve poststroke in subjects (Rand et al., 2010); however, the methodological limitation of this study were pointed out (Cummnig et al., 2012). It is unknown whether these precedence works remain in a report of a functional restitution of the single side of a cognitive function fundamentally, and it is unknown whether these are effective to a memory disorder.

Therefore, in this study, we aimed to examine whether memory function improves because of carrying out a physical activity in a patient who presented a memory disorder after hypoxic encephalopathy. We hypothesized that short-time aerobic exercise increases the memory function of a patient with memory disorder.

\section{Subject}

\subsection{Case}

The patient was a right-handed man; he was 48 years old at the time of onset.

\subsection{History of present illness}

He had a history of idiopathic cardiac hypertrophy for the past seven years. After having fallen while on-duty, the subject was discovered; first-aid conveyance was carried out. After cardiopulmonary arrest was identified, cardiopulmonary resuscitation was performed. His sensorium returned to normal after five days, and he was diagnosed with hypoxic encephalitis. He underwent treatment for the acutestage symptom then he was transferred to the rehabilitation hospital two months after aiming for it.

\subsection{Neurological view}

There is no neurological view that should be mentioned specifically.

\subsection{The computed tomographic scanning of the brain}

Although the presence of phrenatrophia and cerebral edema was not clearly identified, a region with slight high-absorbance was observed in the Gallen sinus venosus and the tentorial sinus (Figure 1).

\subsection{Neuropsychological view}

The result of the neuropsychological tests that were carried out at baseline are shown in Table 1 with the standard and cutoff values for the age group. With regard to Raven's Colored Progressive Matrices (RCPM), which evaluates nonverbal intelligence function, the subject's scores exceeded the cutoff value; however, the scores of the tests that evaluate other cognitive functions, intelligence function, and memory function were much lower than the standard values. Although the subject's attention ability was observed to decline slightly, a decline in executive function was not observed. As measured by Wechsler Adult Intelligence Scale III (WAIS III), which indexes intelligence function, verbal intelligence quotient (VIQ) was 64, performance intelligence quotient (PIQ) was 63, and fullscale intelligence quotient (FIQ) was 61; the score of RCPM were 28 points. As measured by Wechsler Memory Scale Revised (following WMS-R), which 

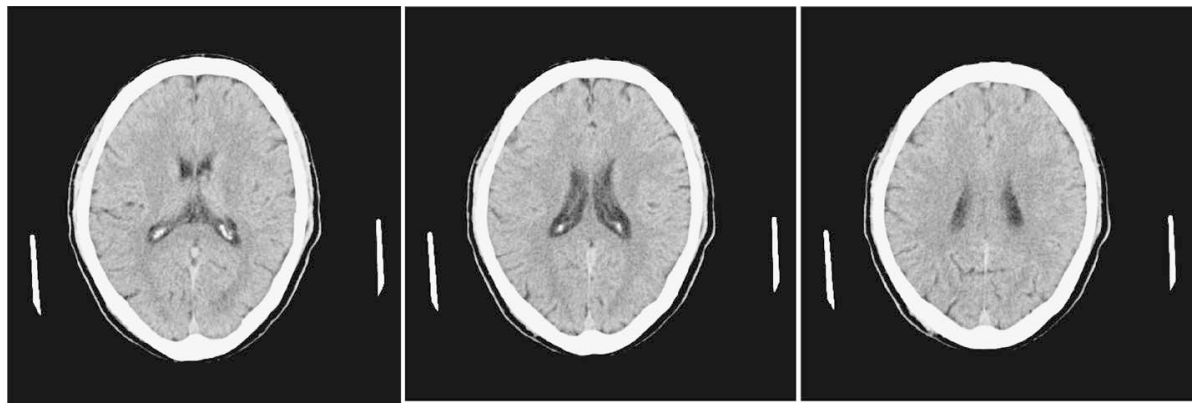

Figure 1 The computed tomographic scanning of the brain of this case.

Table 1 The result of Neuropsychological tests.

\begin{tabular}{|c|c|c|c|c|c|}
\hline & & $\begin{array}{c}\text { Before } \\
\text { Intervention }\end{array}$ & $\begin{array}{c}\text { After } \\
\text { Intervention }\end{array}$ & $\begin{array}{l}\text { The standard } \\
\text { value of a Sub- } \\
\text { ject's age group }\end{array}$ & Cut off score \\
\hline \multicolumn{6}{|l|}{ Intelligence } \\
\hline \multirow[t]{3}{*}{ WAIS-III } & Verbal IQ & 64 & 64 & & \\
\hline & Performance IQ & 63 & 72 & & \\
\hline & Full IQ & 61 & 65 & 102.5 & \\
\hline RCPM & & 28 & 31 & & 24 \\
\hline \multicolumn{6}{|l|}{ Memory } \\
\hline \multirow[t]{5}{*}{ WMS-R } & Verbal & $<50$ & $<50$ & 100.2 & \\
\hline & Visual & 62 & 62 & 100.3 & \\
\hline & General & $<50$ & $<50$ & 100 & \\
\hline & Attention $\cdot$ Concentration & 76 & 87 & 99.9 & \\
\hline & Delayed recall & $<50$ & $<50$ & 100.2 & \\
\hline \multirow[t]{2}{*}{ RBMT } & Standard profile score & 8 & 12 & & $16 / 17$ \\
\hline & Screening score & 4 & 4 & & $7 / 8$ \\
\hline \multirow{2}{*}{$\begin{array}{l}\text { Miyake-shiki memory } \\
\text { retention test }\end{array}$} & Related opposite word & $4-3-4$ & $4-5-6$ & $8.5-9.8-10.0$ & \\
\hline & Not related opposite word & $0-0-0$ & $0-1-0$ & $4.5-7.6-8.5$ & \\
\hline \multicolumn{6}{|l|}{ Executive function } \\
\hline \multirow[t]{3}{*}{ BADS } & Total profile score & 15 & 14 & & 13 (Penumbral) \\
\hline & The standardized score & 85 & 80 & & 75 \\
\hline & $\begin{array}{l}\text { The standization score which carried } \\
\text { out the year-old correction }\end{array}$ & 88 & 83 & & 78 \\
\hline \multicolumn{6}{|l|}{ Attention } \\
\hline SDMT & & 30 & 28 & & 49 \\
\hline
\end{tabular}

WAIS-III: Wechsler Adult Intelligence Scale-III, RCPM: Raven's Colored Progressive Matrices, WMS-R: Wechsler Memory ScaleRevised, RBMT: Rivermead behavioral memory test, BADS: Behavioral Assessment of the Dysexecutive Syndrome, SDMT: Symbol Digit Modalities Test

evaluates the memory function, verbal memory score was lower than 50 , visual memory score was 62 , general memory score was lower than 50 , attention score was 76 , and delayed recall score was lower than 50. With regard to Rivermead behavioral memory test (RBMT), which is used to evaluate everyday memory, the standard profile score were 8 points and the screening score were 4 points. In Miyake-shiki Memory-retention test, the result of relation word was 4 in the first, 3 in the second, 4 in the third, and an opposite word was 0 in the first, 0 in the second, 0 in the third. Symbol Digit Modalities Test (SDMT), which evaluates the divided at- tention and processing speed in Clinical Assessment for Attention (CAT), showed that the subject delivered 30 responses in 90 seconds. Moreover, according to Behavioral Assessment of the Dysexecutive Syndrome (BADS), the total profile score was 15 points, standard score was 85 points, and standard score for the year-old correction was 88 points.

\subsection{The components of rehabilitation}

The subject received each of physical therapy, occupational therapy, and speech-language therapy for about an hour, 5 to 7 times per week. Physical 
therapy included aerobic training, gait training, and step training. Occupational therapy included rangeof-motion training, strength reinforcement training, balance training, and cognitive training. Speechlanguage therapy included cognitive training, which excluded intervention of this study.

\section{Methods}

Single-case experimental design was used (Hacker et al., 1980) in this study. The delayed recall task, in which the subject recalls 3 previously memorized words, and the word fluency task, in which the subject recalls as many words starting with a particular letter as he/she can, were included in this study.

This study was conducted according to the Declaration of Helsinki. The local ethics committee (Department of Rehabilitation Medicine, Nishiyamato Rehabilitation Hospital, Japan) approved this project (protocol number 16). The participant gave written informed consent to participate in this study[0]. Procedures used in this study were in accordance with institutional guidelines.

\subsection{The time of initiation of the trial}

3 months after onset.

\subsection{Experimental tasks}

In the delayed recall task, the memorizing ability of healthy individuals is reported to be 5 or 6 words (Baddeley et al., 1975). Therefore, for conventional memory tasks, such as Hasegawa dementia easy scale-revised (HDS-R) and Mini Mental State Examination (MMSE), 3-word memorization tasks were conducted; we considered it as the pair of every three words at the randomly (Table 2). In the Test of Lexical Processing in Aphasia (Fujita et al., 2000), the vocabulary used was evaluated. The initial sound of the Japanese-syllabary character in a word retrieval task was set from "a" to "ko" in ten trials.

\subsection{Trial procedure}

The study was carried out once a day depending on the time frame assigned to the daily speechlanguage therapy. We used A-B-A study design; it included a baseline phase (A), intervention (aerobic
Table 2 Words used in this study.

\begin{tabular}{cccc}
\hline \multicolumn{4}{c}{ Three words } \\
\hline 1 & Pencil & Teacup & Gauze \\
2 & Thread & Egg & Camera \\
3 & Banana & Newspaper & Sushi \\
4 & Kettle & Pillow & Egg \\
5 & Cup & Ham & Springonion \\
6 & Tempra & Orange & Spoon \\
7 & Pencil & Butter & Carrot \\
8 & Japanese radish & Sake & Match \\
9 & Eraser & Grape & Tomato \\
10 & Strawberry & Candy & Tofu \\
\hline
\end{tabular}

exercise) phase (B), and washout phase (A). The duration of each phase was ten days (Figure 2).

3.3.1. Baseline phase and washout phase: Delayed recall tasks were carried out according to the following protocol. We showed a character card containing three words to a subject and let him memorize the words. To check whether he remembers them, we asked him to repeat the words immediately. Then, a freely speech and a desk task were conducted after 15 minutes. After 15 minutes, the patient was asked to recall the 3 words. Furthermore, word fluency tasks were conducted during the speech-language therapy session.

3.3.2. Intervention (aerobic exercise) phase: After the subject to memorizes the words on a character card, as described above. To confirm whether he has memorized them, we asked him to repeat the words immediately. Next, we let him carry out continuous physical exercise for 15 minutes using a bicycle ergometer (COMBI AEROBIKE 2100R). We set the intensity at $50 \mathrm{~W}$, which is low, because the subject presented bradycardia, which is a symptom of heart disease and the facilitate of the bradycardia by a physical exercise stress was seen. We set the exercise intensity range as 11 (easy)-13 (slightly difficult) points according to the Borg scale (Borg, 1982) presented by the medical doctor. After 15 minutes, we let him recall the 3 memorized words and carry out word fluency tasks. Furthermore, we measured the heart rate (HR) of the subject just before, in the middle of, and immediately after the physical exercise, and we recorded the value displayed on the HR monitoring device. Additionally, the tester checked whether the subject exercised continuously for a certain time during a measurement of HR. 


\begin{tabular}{|c|c|c|}
\hline $\begin{array}{c}\text { Three-word memory- } \\
\text { storage task }\end{array}$ & $\begin{array}{c}\text { Three-word memory- } \\
\text { storage task }\end{array}$ & $\begin{array}{c}\text { Three-word memory- } \\
\text { storage task }\end{array}$ \\
\hline $\begin{array}{c}\text { Free talk and } \\
\text { paper- and-pencil task }\end{array}$ & $\begin{array}{c}\text { bicycle-ergometer physical } \\
\text { exercise }\end{array}$ & $\begin{array}{c}\text { Free talk and } \\
\text { paper- and-pencil task }\end{array}$ \\
\hline
\end{tabular}

\begin{tabular}{|l|c|c|}
\hline $\begin{array}{c}\text { Three-word } \\
\text { delayed recall task }\end{array}$ & $\begin{array}{c}\text { Three-word } \\
\text { delayed recall task }\end{array}$ & $\begin{array}{c}\text { Three-word } \\
\text { delayed recall task }\end{array}$ \\
\hline Word fluency task & Word fluency task & Word fluency task \\
\hline
\end{tabular}

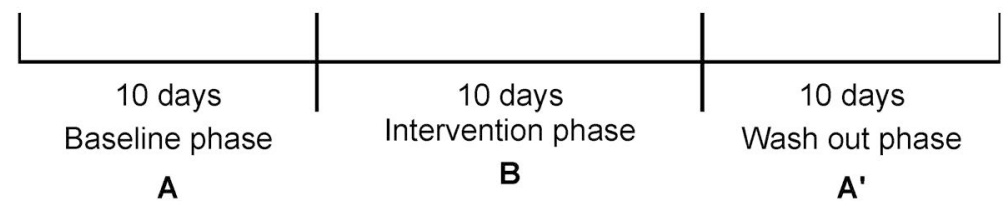

Figure 2 Protocol of this study

\subsection{Contents of assessment}

We assessed the number of words that were correctly recalled (the maximum number is three) in delayed recall task and the number of retrievals in word fluency task (with no maximum number of correct answers). Moreover, neuropsychological tests were carried out before and after the trial.

\subsection{Statistical analysis}

The datum of each variety was represented as mean and standard deviation. We conducted the variance analysis by repetitive measurement (repeated measure ANOVA); the number of words recalled correctly in the delayed recall task, the differences in the results of the word fluency task among the baseline phase, intervention phase, and washout phase, and the difference among the HRs that were measured before, in the middle of, and immediately after aerobic exercise (intervention phase) were evaluated. We used IBM SPSS software version 25 for the statistical analysis, and we set the significance level at $5 \%$.

\section{Results}

\subsection{Delayed recall task}

The results of delayed recall task are shown in Figure 3. In the baseline phase, the subject was able to recall zero words among the three memorized words (10-day average $=0 \pm 0)$. On the contrary, in the intervention phase, the number of words recalled correctly was 1 on the first day. Although this number decreased to zero on the second day, it increased to three on the third day. This ability to recall all three words was observed for 5 days henceforth; however, it decreased to one word on the eighth day. On the last two days the patient was able to recall three word (10-day average $=2.3 \pm 1.1$ words). In the washout phase, the subject was able to recall zero words on the first eight days. Although the subject was able to recall one word on the ninth day, it decreased to zero words on the tenth day (10-day average $=0.1 \pm 0.3$ words). The number of mean correct answers was significantly different among phases as demonstrated by repeated measure ANOVA $(F(2,18)=37.098, p<0.0001)$ Significant differences were observed (multiple comparison by Tukey's test) between the baseline phase and intervention phase $(\mathrm{p}<0.0001)$ and between the intervention phase and withdrawal phase $(p<$ $0.0001)$.

\subsection{Word fluency task}

The results of word fluency task are shown in Figure 4. He went up to the 6th time at four words from two words whose results of a baseline term are the 1 st time, he fell to an octavus time renewedly at two words. The average number of words retrieved was $2.7 \pm 0.9$. In the intervention phase, the number of words retrieved range from 0 to 4 after aerobic 


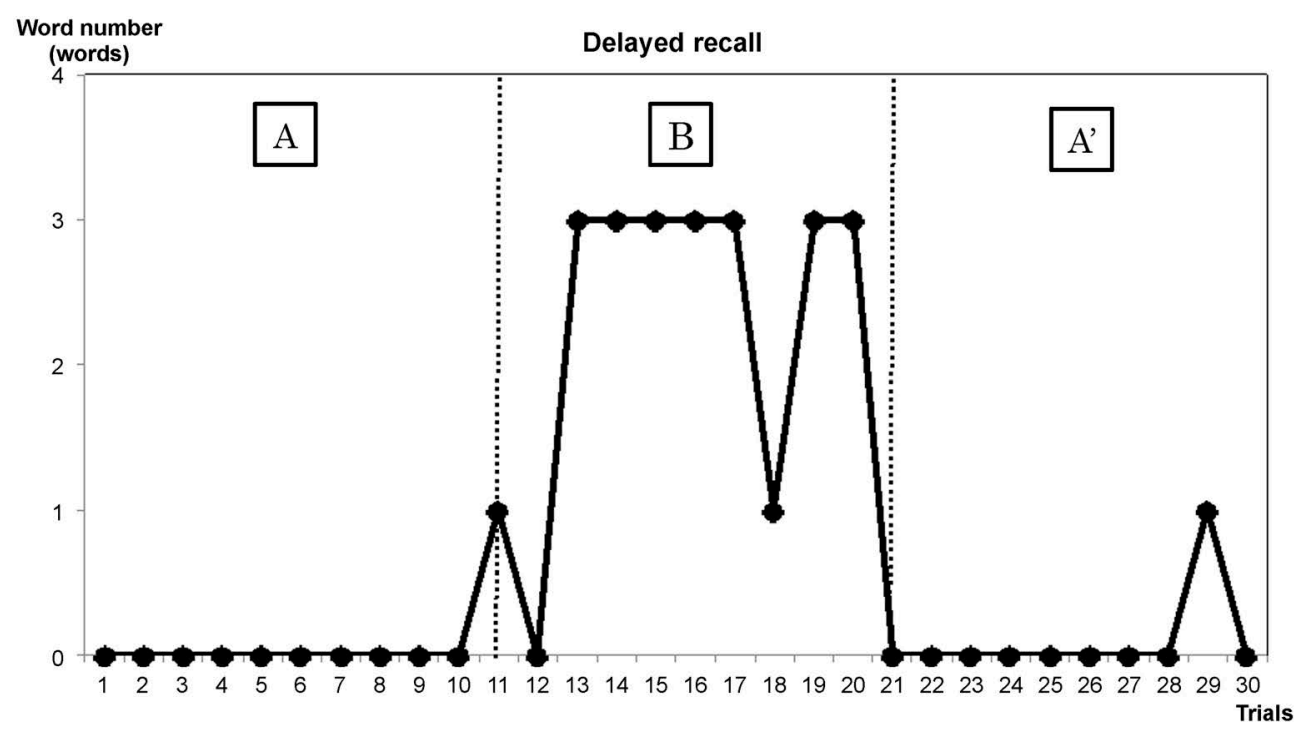

Figure 3 The result of delayed recall task

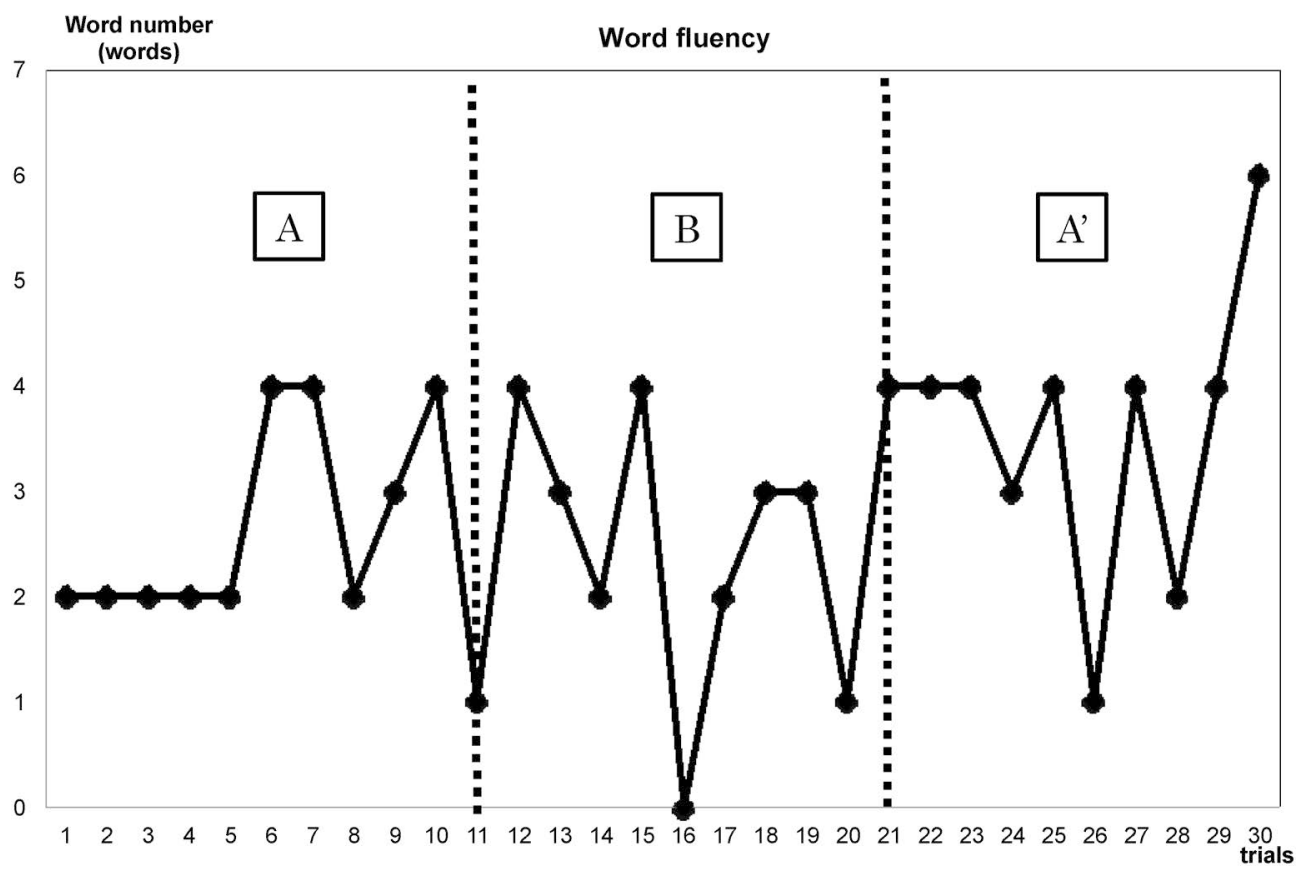

Figure 4 The result of word fluency task

exercise using a bicycle ergometer (10-day average $=$ $2.3 \pm 1.3$ words). In the washout phase, he went up the result of 1-4 words to six words to a repeatedly and the 10th time (10-day average $=3.6 \pm 1.3$ words). Repeated measure ANOVA showed no significant difference $(\mathrm{F}(2,18)=2.739, \mathrm{p}=0.092)$.

\subsection{Heart rate during the intervention phase}

In the intervention phase, we measured the $\mathrm{HR}$ at before, in the middle of (7.5-minute timepoint), and immediately after (15-minute timepoint) cycling; thereafter, we could obtain seven times outcomes of 10 times. As shown in Figure 5, the average HR before, in the middle of, and immediately after aerobic exercise was $48.3 \pm 3.6 \mathrm{bpm}, 61.3 \pm 9.8 \mathrm{bpm}$, and $44.9 \pm 2.5 \mathrm{bpm}$, respectively. Repeated measure ANOVA showed a significant main effect $(F(2,12)$ $=14.173, \mathrm{p}=0.001)$. A significant difference was observed between the HRs recorded in the middle of and immediately after aerobic exercise (multiple comparison by Tukey's test; $\mathrm{p}=0.001$ ). 


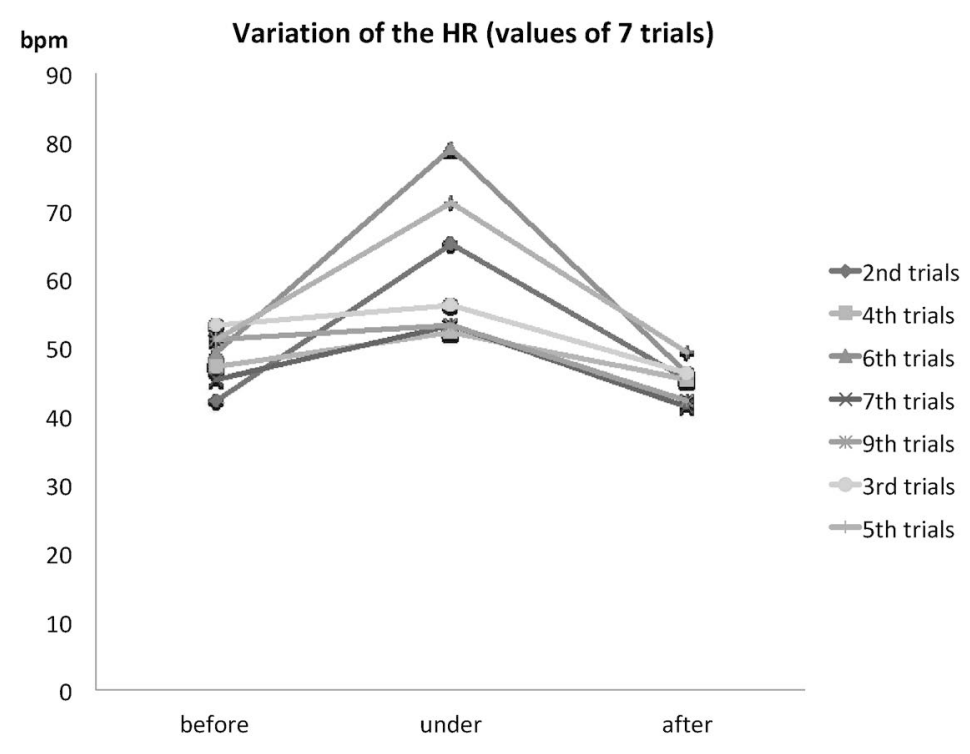

Figure 5 The changes of heart rate

\subsection{Neuropsychological reevaluation after the ter- mination of the trial}

The results of neuropsychological reevaluation after the termination of the 30-day trial are shown in Table 1. VIQ was 64, PIQ was 72, and FIQ was 65 as measured by WAIS-III, which evaluates the intelligence; these results indicate an improvement in cognition. As measure by WMS-R, a memory test, verbal memory score was 50 or lower, visual memory score was 62 , general memory score was 50 or lower, attention and concentration score was 87 , and delayed recall score was 50 or lower; attention and concentration score was higher, compared to that at the time of hospitalization. The results of other items evaluating memory ability showed no variation, compared to those at the time of hospitalization. On the contrary, as measured by RBMT, which evaluates everyday memory, standard profile score was 12 points, and screening score was 4 points; these scores indicated a slight improvement. Additionally, the results of Miyake-shiki Memoryretention test showed improvement; the result of related word was 4 in the first, 5 in the second, 6 in the third, an opposite word was 0 in the first, 1 in the second, 0 in the third. With regard to Behavioral Assessment of the Dysexecutive Syndrome (BADS), the total profile score was 14 points, standard score were 80 points, and standard score by age and overall classification by age were 83 points; Symbol Digit Modalities Test (SDMT) in Clinical Assessment for Attention (CAT), showed that the subject delivered 28 responses in 90 seconds, indicating a decreased performance.

\section{Discussion}

To verify whether a restoration of a memory is brought about by physical exercise in the subject who presented memory disorder after hypoxic encephalitis in this study, the results of delayed recall task and word fluency task were examined at the baseline phase, intervention (aerobic exercise) phase, and washout phase.

First, with regard to the delayed recall task, the task of remembering three words was difficult for the subject in the baseline phase. However, in the intervention phase, after carrying out physical activity on a bicycle ergometer for 15 minutes, the mean number of words recalled correctly was $2.3 \pm$ 1.1. Furthermore, in the washout phase, the subject was able to recall zero words on all days, with the exception of day 9 - on which one word was recalled; significant difference was observed in the number of times each term was recalled, we made the variation of the result by involvement into the obvious. The results of word fluency task were influenced by bicycle ergometer activity. The HR at the end of cycling $(44.9 \pm 2.5 \mathrm{bpm})$ was lower than that at the beginning of cycling $(48.3 \pm 3.6 \mathrm{bpm})$, because the subject had a heart disease and sthenia of bradycardia was considered to caused by the stress of physical exercise. In addition, about this case being the involvement three months after a 
sideration, although the existence of the effect of a spontaneous cure is suspected as for it, the result whose result improved as compared with the word retrieval task carried out to the synchronously. It is possible to realize it as the immediately effect of a simple body activity involvement.

With regard to the word fluency task, as shown in Figure 4, although an increase in the number of word retrieved from two words to four words was observed in the baseline phase, he retrieved words repeatedly from zero to maximum four words at an intervention phase, he could not retrieved more than 4 words. In the washout phase, after retrieval of continued four words, the number of his retrieve became low from 1 word, and 2 words, and finally he could retrieve 6 words. The trouble of word fluency has been reported to be due to frontal lobe damage (Saito. et al., 1992). However, the trouble with word retrieval is said to be clear in the frontal lobe damage; it is thought to be due to a cerebral lesion. In the word fluency task, although aerobic exercise may have influenced the improvement of word retrieval, the result was not sufficiently effective. Retrieval of six words possibly reflected the highest learning effect of aerobic exercise.

Furthermore, the neuropsychological reevaluation, which was carried out after the completion of the trial, showed that PIQ (measured using WAISIII) improved from 63 points to 72 points and that the scores representing nonverbal intelligence improved from 28 to 31 (measured using RCPM). Although the attention and concentration scores, which were measured by using WMS-R, improved from 76 to 87 , no improvement was observed with regard to the items related to memory ability. However, standard profile scores, which were measured by RBMT, improved from 8 to 12 . The related words score improved from 4-3-4 to 4-5-6, and the non-related words score improved from 0-0-0 to 0-1-0 in Miyake-shiki Memory-retention test. In BADS, which measures executive function, a slight decline in the total profile score (from 15 to 14), standard score (from 85 to 80 ), and standard score for the year-old correction (from 88 to 83 ) was observed. The responses delivered by the subject in 90 seconds declined from 30 to 28 (SDMT). Therefore, the improvement in intelligence function, attention and concentration, and memory ability in an everyday life situation, indicates an improvement in cognitive function, excluding direct memory ability.
However, restitution of memory was not greatly achieved as demonstrated by the evaluation of memory function itself; even if acute effect of memory function is achieved, by the involvement for about one month, fundamental restitution of memory and synthetic cognitive function are not achieved. However, neuropsychological evaluation after intervention for a relatively longer period is necessary in the future.

In this study I found that the delayed recall task improved while the word fluency task did not improve in the two cognitive tasks after intervention of aerobic training. Regarding improvement in the cognitive function by aerobic training, factors of locomotor system, the neural system, and the ciruculatory-organ system are involved (Ahlskog et al., 2011, Kirk-Sanchez \& McGough, 2014). In this study, the factor that brought about the restitution of memory ability in a patient with brain damage cause by hypoxic encephalitis was difficult to determine; however, increased HR, which was observed after 15 minutes of bicycle ergometer activity, is possibly a factor that influenced the restitution of memory ability (Figure 5). In healthy participants, an increase in HR by physical exercise has been confirmed to improve attention, concentration, cognitive function (Albinet et al., 2010, Luque-Casado et al., 2016). In this study although the participant had hypoxic encephalopathy, it was suggested that he could gain the attention to memorize and retain the memorized word by exercise-induced HR rise as reported in the previous studies. Furthermore, in the delayed recall task, the subject forgot the words in the baseline phase, which involved physical inactivity. Physical inactivity is reportedly associated with cognitive decline (Rhodes et al., 2012, Hamer and Stamatakis., 2015); it is considered to be a reflect result in the same tendency to have used by this study, although it is not a habitual sitting behavioral. With regard to word fluency task, it is thought that the subject could not retrieve a word newly despite the ability of physical exercise to stimulate the retention of a shown word. In the brain, the Papez circuit, which involves the hippocampus, manages the memory function and the frontal lobe manages the word fluency power. A report by Erickson and colleagues (2011) shows that the volume of the hippocampus increases with aerobic exercise; association between physical exercise and activity of hippocampal neurons exist. 
However, in this study, it is difficult to understand in this one intervention study that performing aerobic exercise for 15 minutes results in the restitution of memory is a result of improvements of memory function and hippocampus function. To clarify the influence these function, it is important to confirm the change of memory function in the intervention with sufficient duration such as the preceding study, and to measure the brain function. However, in baseline phase, the 15 -minute period after memorization involved interferences such as conversation and paper and pencil task in sitting posture; this may have affected the subject's performance in delayed recall task. In this regard, it is necessary to set up a protocol that is controlled for interference to facilitate elucidation of the reason for difficulty in performing delayed recall task in the baseline phase.

As a result of including an aerobic exercise as an intervention, we obtained a result that reflects realtime improvement in memory ability. However, in the future, it is necessary to verify whether only aerobic training has a good effect or other exercise also have good effects involved resistance training. It is desirable to verify the effect of frontal-lobe function not only on memory function but also on executive function or intelligence function in addition to processing speed as a cognitive function. Furthermore, the effect verified by a single-case study is limited. Therefore, verifying this effect by evaluating a higher number of cases is necessary in the future. We believe that elucidating the effect of physical exercise and physical activity on cognitive function would provide an effective intervention approach for the improvement of cognitive function.

\section{Conclusion}

We examined the result of the word fluency and delayed recall tasks in baseline phase, intervention (aerobic training) phase, and washout phase, to evaluate whether the subjects with impaired memory function would recover by aerobic-activity intervention in this study. In the intervention phase, improvement was not observed in the word fluency task; however, there was an improvement in the delayed recall task. Therefore, an aerobic exercise is considered to improve the probability of real-time restitution of memory.

\section{Acknowledgment}

We express our gratitude to Dr. Shigeru Morimoto, Nishiyamato Rehabilitation Hospital, who was instructed to decipher the brain image in this research, and we thank the patient who cooperated in the experiment. Part of this research was supported by the 44th Daiwa Securities Health Foundation.

\section{References}

Aarsland, D., Sardahaee, F. S., Anderssen, S., and Ballard, C. (2010). Is physical activity a potential preventive factor for vascular dementia? A systematic review. Aging Ment. Health., 14(4): 386-95.

Ahlskog, J. E., Geda, Y. E., Graff-Randford, N. R., Petersen, R. C., and Proc, M. C. (2011). Physical exercise as a preventive or disease-modifying treatment of dementia and brain aging. Mayo. Clin. Proc., 86(9): 876-84.

Albinet, C. T., Boucard, G., Bouquet, C. A., and Audiffren, M. (2010). Increased heart rate variability and executive performance after aerobic training in the elderly. Eur. J. Appl. Physiol., 109(4): 617-24.

Baddeley, A. D., Thomson, N., and Buchanan, M. (1975). Word length and the structure of short-term memory. J. Verbal Learning Verbal Behav., 14(6): 575-589.

Borg, G. A. (1982). Psychophysical bases of perceived exertion. Med. Sci. Sports Exerc., 14(5): 377-381.

Cicerone, K. D., Langenbahn, D. M., Braden, C., Malec, J. F., Kalmar, K., Fraas, M., Felicetti, T., Laatsch, L., Harley, J. P., Bergquist, T., Azulay, J., Cantor, J., and Ashman, T. (2011). Evidence-based cognitive rehabilitation: updated review of the literature from 2003 through 2008. Arch. Phys. Med. Rehabil., 92(4): 519-30.

Colcombe, S. J., Kramer, A. F., Erickson, K. I., Scalf, P., McAuley, E., Cohen, N. J., Webb, A., Jerome, G. J., Marquez, D. X., and Elavsky, S. (2004). Cardiovascular fitness, cortical plasticity, and aging. Proc. Natl. Acad. Sci. U.S.A., 101(9): 3316-3321.

Cumming, T. B., Tyedin, K., Churilov, L., Morris, M. E., and Bernhardt, J. (2012). The effect of physical activity on cognitive function after stroke: a systematic review. Int. psychogeriatr., 24(4): 557-567.

Doornhein, K. and deHaan, E. H. F. (1998). Cognitive training for memory deficits in stroke patients. Neuropsychol. Rehabil., 8(4): 393-400.

Erickson, K. I., Voss, M. W., Prakash, R. S., Basak, C., Szabo, A., Chaddock, L., Kim, J. S., Heo, S., Alves, H., White, S. M., Wojcicki, T. R., Mailey, E., Vieira, V. J., Martin, S. A., Pence, B. D., Woods, J. A., McAuley, E., and Kramer, A. F. (2011). Exercise training increase size of hippocampus and improves memory. Proc. Natl. Acad. Sci. U.S.A., 108: 3017-3022.

Fujita, I., Monoi, H., Okudaira, N., Ueda, M., Ono, K., Shimogaki, Y., Fujiwara, Y., Furuya, F., and Sasanuma, S. (2000). Shitsugosho goikensano kaihatsu [Developing a Test of Lexical Processing in Aphasia (TLPA)]. Jpn. J. Logop. Phoniatr, 41(2): 179-202. (in Japanese)

Hacker, B. (1980). Single subject research strategies in occupational therapy, part 1. Am. J. Occup. Ther., 34 (2): 103-108.

Hamer, M. and Stamatakis, E. (2014). Prospective study of sedentary behavior, risk of depression, and cognitive impairment. Med. Sci. Sports. Exerc., 46(4): 718-23.

Kirk-Sanchez, N. J. and McGough, E. L. (2014). Physical exercise and cognitive performance in the elderly: current per- 
spectives. Clin. Interv. Aging, 9: 51-62.

Kojima, M. and Nagano, A. (2018). The Association between Total Energy Expenditure and Cognitive Function in Convalescent Patients with Cerebrovascular Disease. Jpn. J. Phys. Educ. Health Sport Sci., 63: 77-85. (in Japanese)

Kramer, A. F., Erickson, K. I., and Colcombe, S. J. (2006). Exercise, cognition, and the aging brain. J. Appl. Physiol., 101: $1237-1242$.

Luque-Casado, A., Perales, J. C., Cárdenas, D., and Sanabria, D. (2015). Heart rate variability and cognitive processing: the autonomic response to task demands. Biol. Psychol., 113: 83-90.

Mimura, M. and Komatsu, S. (2003). Kiokusyougaino rihabiriteesyonnno arikata [Theoretical frameworks of memory rehabilitation]. High Brain Funct. Res., 23(3): 181-190. (in Japanese)

Quaney, B. M., Boyd, L. A., McDowd, J. M., Zahner, L. H., He, J., Mayo, M. S., and Macko, R. F. (2009). Aerobic exercise improves cognition and motor function poststroke. Neurorehabil. Neural. Repair., 23(9): 879-885.

Rand, D., Eng, J. J., Liu-Ambrose, T., and Tawashy, A. E. (2010). Feasibility of a 6-month exercise and recreation program to improve executive functioning and memory of individuals with chronic stroke. Neurorehabil. Neural. Repair, 24(8): 722-729.

Rhodes, R. E., Mark, R. S., and Temmel, C. P. (2012). Adult sedentary behavior: a systematic review. Am. J. Prev. Med., 42(3): e3-28.

Saito, H., Kato, M., Kashima, H., Asai, M., and Hosaki, H. (1992). Zentoyosonsyoto Word Fluency - tokuni yokuseisyogaitono kanrenni tsuite [Effects of disinhibition on performance of the Word Fluency Test in patients with frontal lesions]. High Brain Funct. Res., 12(3): 223-231. (in Japanese)

Smith, P. J., Blumenthal, J. A., Hoffman, B. M., Cooper, H., Strauman, T. A., Welsh-Bohmer, K., Browndyke, J. N., and Sherwood, A. (2010). Aerobic exercise and neurocognitive performance: a meta-analytic review of randomized controlled trials. Psychosom. Med., 72(3): 239-252.

Tatemichi, T. K., Desmond, D. W., Stern, Y., Paik, M., Sano, M., and Bagiella, E. (1994). Cognitive impairment after stroke: frequency, patterns and relationship to functional abilities. J. Neurol. Neurosurg. Psychiatry, 57: 189-98.

Watamori, T. and Honda, R. (2005). Kiokusyougaino rihabiriteesyonn-sono-gutaitekihouhou [Memory rehabilitation - the concrete methods-]. Jpn. J. Rehabil. Med., 42: 313-319. (in Japanese)

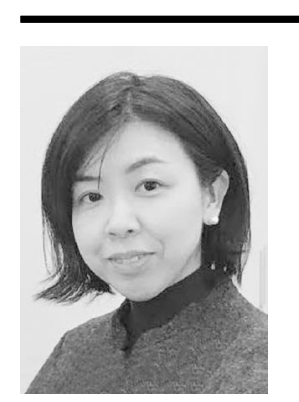

Name:

Maki Kojima

Affiliation:

Department of Rehabilitation Medicine, Nishiyamato Rehabilitation Hospital

\section{Address:}

3-2-2 Sasayuri-dai, Kanmaki-cho, Kitakatsuragi-gun, Nara 639-0218, Japan

\section{Brief Biographical History:}

2009-present: Department of Rehabilitation Medicine, Nishiyamato Rehabilitation Hospital

2016-2019: Graduate School of Sport and Health Science, Ritsumeikan University, Shiga, Japan (Ph. D. program).

2009-2011: Kobe University Graduate School of Health Sciences, Kobe, Japan (master program).

2004 received speech language pathologist

\section{Main Works:}

-Kojima, M., and Nagano, A. (2019). Assessment of physical activity and cognitive function and their potential correlation in convalescent patients of cerebrovascular disease. Sci. Rep. 9(1): 3782.

-Kojima, M., Okahashi, S., Luo, Z., Nagano, A., Sakai, H., and Seki, K. (2016). Development of a Virtual Reality-based comprehends cognitive functions test for people with aphasia. High Brain Funct. Res., 36(2): 296-303. (in Japanese)

-Kojima, M., Okahashi, S., Tanemura, R., Nagano, A., Luo, Z., and Seki, K. (2012). A trial of virtual reality-based cognitive assessment for aphasic patients. Jpn. J. Speech Lang. Hear. Res. 9(2): 80-88. (in Japanese)

Membership in Learned Societies:

-The Japanese Association of Speech-Language-Hearing Therapists

- Japan Society for Higher Brain Dysfunction

-The Japanese Society of Dysphagia Rehabilitation 